\title{
METHODOLOGICAL ASPECTS OF EVALUATION OF STATE BUDGE! PROGRAMMES
}

\author{
Juozas Bivainis $^{1}$, Algirdas Butkevičius ${ }^{2}$ \\ ${ }^{1}$ Dept of Social Economics and Management, Vilnius Gediminas Technical University, Sauletekio ai. 11, \\ LT-2040 Vilnius, Lithuania. E-mail: vvfsevk@vv.vtu.lt \\ ${ }^{2}$ Budget and Finance Committee, Parliament of Republic of Lithuania, Gedimino av. 53, \\ LT-2002 VilniusXithuania. E-mail: albutk@lrs.lt
}

Received: 6 January, 2003

\begin{abstract}
The paper deals with the reasonability of allocation of public financial resources. It presents an integrated evaluation model applicable to budget programmes and capable of deepening the comprehensiveness as well as the objectivity of programme evaluation aimed at benchmarking. The nucleus of the model consists of four components: evaluation of relevance, evaluation of efficiency, evaluation of fitness, and the synthesis of the said evaluations. The contents and main methodological aspects of each component are described.
\end{abstract}

Keywords: state budget, expenditure, planning, integrated evaluation, methods.

\section{Introduction}

Preparation and execution of the national budget is one of the most significant areas of public administration, which is easy to prove by mentioning that a considerable part of the national product is redistributed through the budget (it varies among different states from 20 to 50 per cent of the GDP [1]).

The procedure of preparation and execution of the state budget reminds of a chain that starts with forecasting certain macroeconomic indicators and ends with auditing how the financial resources were used and targeted control of institutions. The key position in the chain is taken by the task of allocation [2-4]. Only rational allocation of financial resources is able to precondition efficient use of scarce resources the shortage of which is typical to all countries - small or large, economically poor or rich. From a different angle, the allocation of public financial resources may be treated as a means to fulfil priority tasks of the state in accordance with government programmes and other programme documents and to invigorate the activities in certain areas $[2,5,6]$.
The governments of all the world countries, some more actively and some in more passive manner, try to find ways to improve the allocation of financial resources. The USA came up with the most outstanding initiatives in as early as 1965 when it started experimenting with the application of programme planning principles to the planning of budget expenditure [6].

The recent 15 years have produced the best results of the endeavour. There were numerous factors that facilitated success two of which need to be mentioned specifically because of their significance. One is political and economic reforms in Central and Eastern Europe that shaped the need not only to take over the best practice of other countries but to search for innovative solutions as well, and the other is new information technologies that created preconditions for more efficient application of methods with better tools.

Certain positive changes in the allocation of public financial resources are evident and indisputable. For instance, all EU candidate countries have fundamentally reformed their expenditure management [2] 
bringing it in line with the Community requirements, a multi-year budgeting (for a medium-term period) has become a rule in OECD countries [6], budgeting rests on the principle of publicity provided with the help of the latest information technologies in many countries [7], etc. More advanced and more reasonable tools, as well as more rapid ways of allocating public financial resources are being sought [2, 8, 9]. Lack of constructive solutions - absence of rules of allocating financial resources, doubtful or unreasonable criteria or their neglect have encouraged us to undertake a study aiming at developing principles enabling to assess budget programmes, which would allow each programme (its alternative) to be comprehensively and most objectively evaluated in terms of its efficiency. The goal set has determined the focus of the study allocation of public financial resources.

The study made good use of analytical and empirical methodologies and synthesis, analogue, benchmarking, and logical abstraction methods

\section{Approach - integrated evaluation}

There is no doubt that the method of budget expenditure planning has a direct impact on the result of resource allocation. To this end the principle of programme budgeting is now widely advocated $[2,6,9]$. A generalised review of different approaches and recent reforms in the preparation of the state budget suggests a conclusion that the method of programme budgeting should be considered as a progressive and suitable way that has a potential for the future. More frequent and more serious discussions now centre on the degree of its applicability - should it be applied to the allocation of all the financial resources or in certain areas only $[1,6]$. Anyway, taking on board the programme budgeting method for expenditure planning in its entirety or part, budget programmes (draft programmes, to be exact) are the components in respect to which decisions on financial resource allocation are taken as a relatively large share of financial resources is allocated to programmes.

The challenge, therefore, is to be able to, as objectively as possible, go through a screening exercise and decide whether and how many financial resources should be allocated to a particular programme. More precisely, clear criteria or even algorithms for programme evaluation are needed. Without diminishing the significance of a human factor in decision-making and with due regard to a political aspect, which becomes particularly expressed in the final stages of budget debates, the evaluation of budget programmes should be based on uniform criteria and uniform rules of their application to ensure objectivity. The authors interested in the developments in this field $[2,6,7]$ emphasise the latter, and even though they all agree about its significance, the choice of corresponding tools in current practices is very limited. One can find reasoning and advice on the methodological aspects of dealing with the challenge in numerous publications. The practice of expenditure programme evaluation by the European Union seems to be the most systematic package of recommendations $[10,11]$. It covers programme evaluation at different stages of programme preparation and implementation. The recommendations, unfortunately, are not detailed to the level of quantitative appraisal, thus comes the gap absence of quantitative appraisal instruments, not to mention integrated quantitative appraisal systems.

Some support informal unregulated evaluation of budget programmes, others, though, are of the view that regulation is a must in this field, while evaluation must be conducted in compliance with statutory methodologies. Both approaches have strengths and weaknesses. Regulation reduces freedom of choice, hence scope of creativity in this as well as any other area, and in view of the subject matter under consideration and bearing in mind exceptionally wide spectrum of alternatives (of programmes and the circumstances under which they are implemented), their scope, contents, composition, combinations and other features, unification of evaluation reduces possibilities of objective evaluation. Nevertheless, noting pros and cons wc believe that in respect of one of the key evaluation criteria - benchmarking - methodological guidance in budget programme evaluation might be seen as a necessary attribute. However this does not exclude another requirement - flexibility and universality, combined with possible benchmarking.

The analysis of different studies undertaken in this field $[6,6,10,11]$ and conclusions drawn from several years experience accumulated in preparing the national budget [1] serve as a basis for proposing a system of budget programme evaluation resting on an integrated evaluation method (Fig 1). The system is comprised of four major evaluation components: programme relevance, programme efficiency, programme fitness and integrated evaluation of each programme. The first three components may be used for evaluation of budget programmes from certain perspectives, while the fourth attempts at summarising the results of the three and obtaining integrated evaluation of a programme, which in its turn makes programmes comparable. We propose integrated evaluation because, in our opinion, it is applicable to every new, i.e. draft programme of the budget. While the method of evalu- 


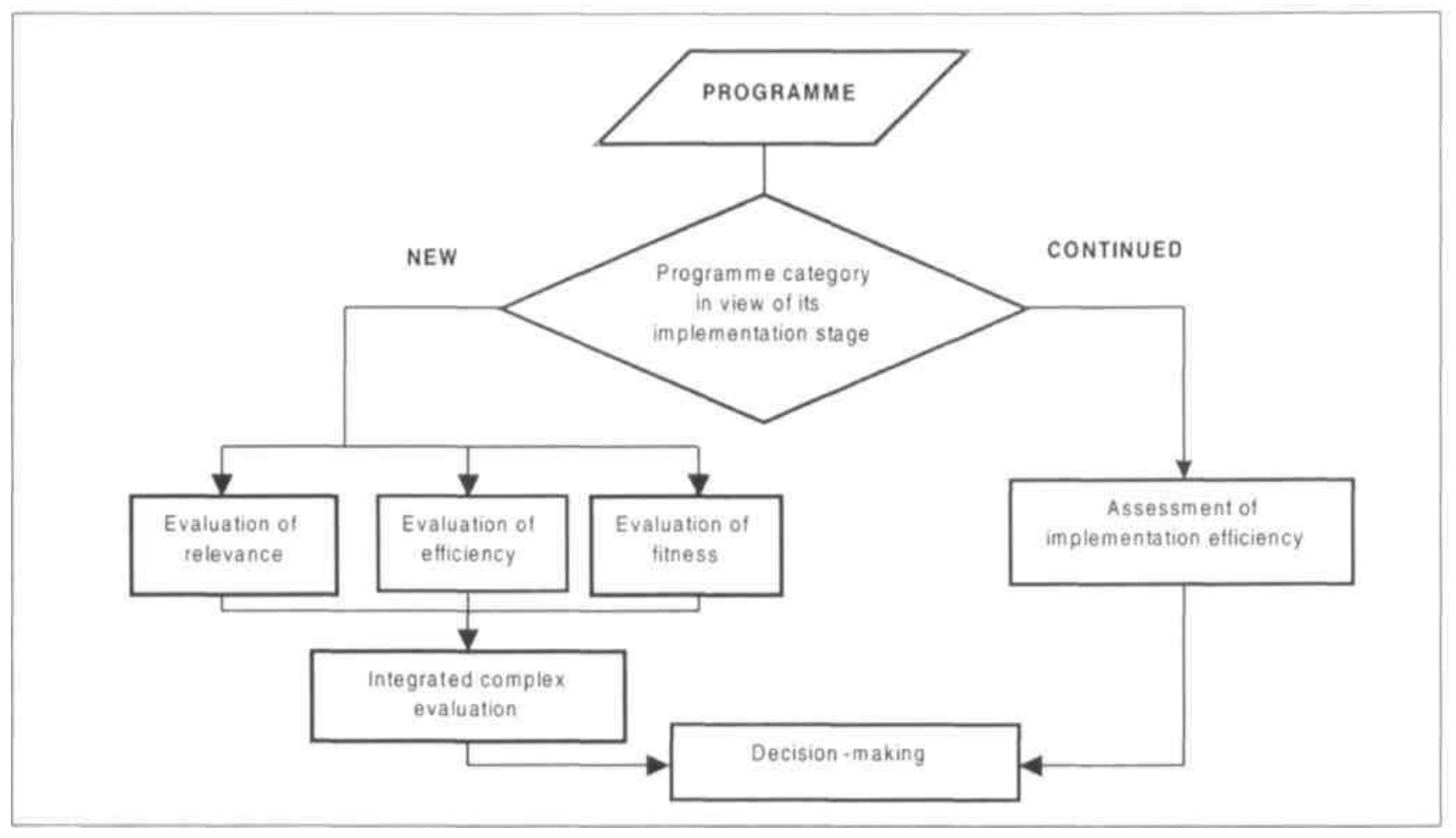

Fig 1. Scheme of budget programme evaluation

ation of multi-year programmes by their performance (efficiency) in previous years might be more precise (less costly and less relative).

\section{Evaluation of programme relevance}

Doing an exercise of integrated evaluation of budget programmes, the sequence in which components are applied is not important and does not essentially effect the decision, on the other hand, though, starting from the component of relevance evaluation may be less labour extensive because of the following two reasons. First, costs of programme relevance evaluation are considerably lower than those of programme efficiency evaluation. Second, results of this component (integrated relevance indicator) suggest whether it is worthwhile proceeding with other evaluation components. For programme relevance evaluation, wc propose a technology (Fig 2) that preconditions two key evaluation tasks: evaluation of different programme aspects and generalised quantitative programme evaluation.

The scheme (Fig 2) presents a list of partial evaluation criteria compiled resorting to experience and should be treated as no more that a recommended procedure. With changing factors of political, social and economic environment, the list should be regularly revised. Its revision should be guided by two most important requirements:

1) Consistency of the list, i.e. one list for all the programmes of a budget;

2) Relative significance of criteria has to meet the following terms:

$$
0 \leq r_{i} \leq 1, \quad \sum_{i=1}^{n} r_{i}=1, \quad i=1,2, \ldots n \text {. }
$$

where: $r$ - relative significance of criteria (relative value in fractions of a unit); $i$ - criterion index.

There are no theoretical restrictions as to the use of different ranges in expert valuation (opinion), for example,relative fractions of a unit, points, probabilities. Priority still should be given to a uniform evaluation range as having two advantages: it offers a benchmark for experts applying different criteria for programme evaluation; it offers a simplified way of evaluating programme relevance according to each criteria. In seeking for a uniform benchmark a line must be drawn because objectivity of evaluation must not suffer at the expense of uniformity.

Evaluation of programme relevance according to each criterion is a restatement of expert valuations (processed expert opinions) in a unified system, i.e. calculation of values of partial relevance indicators. Elementary algorithms reflecting a ratio of criteria ex- 


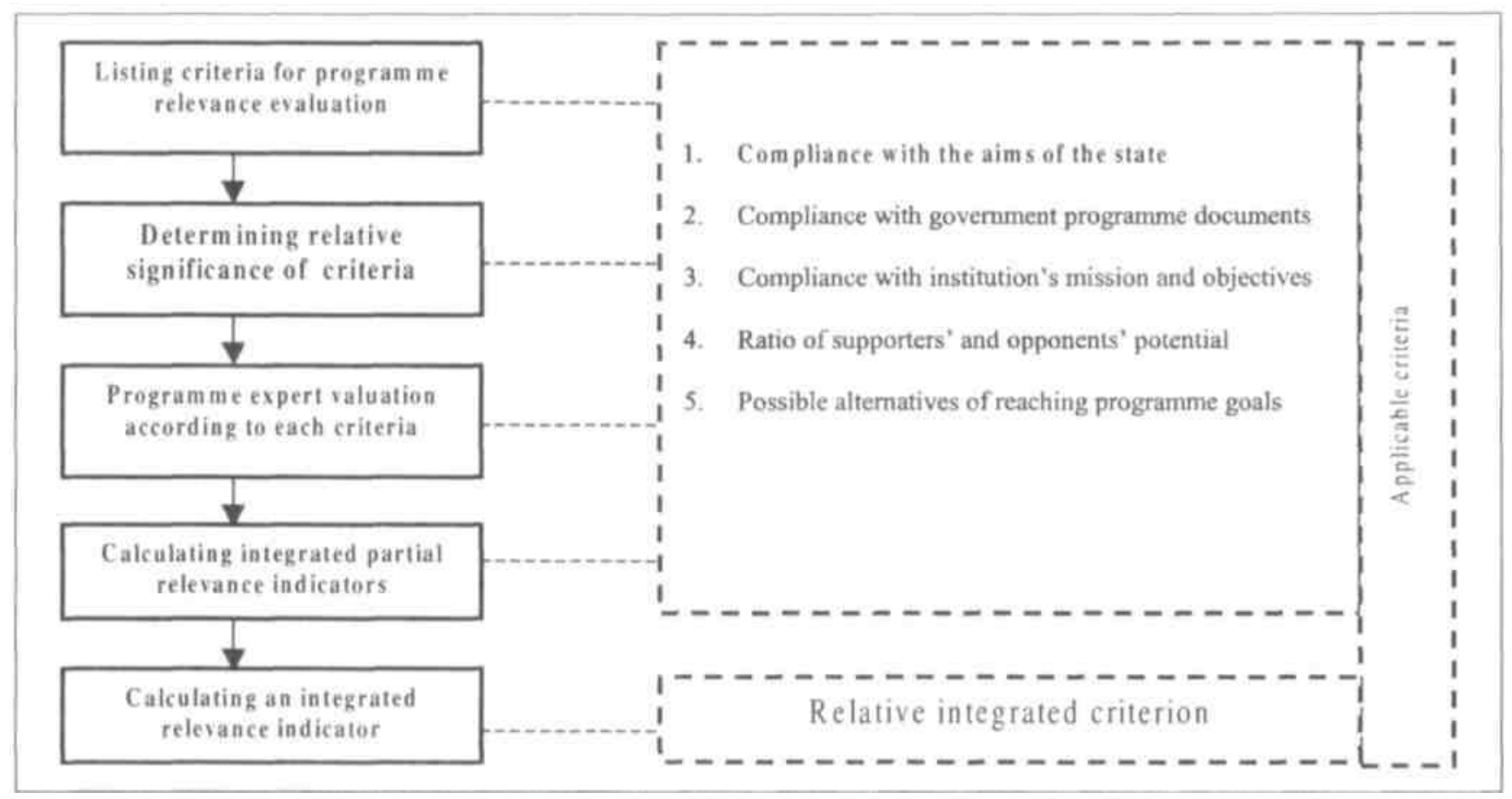

Fig 2. Scheme of programme relevance evaluation

pert value to criteria value in a unified system should be used:

$$
v_{i}=\alpha_{i} v_{i}^{\prime}
$$

where: $v^{\prime}$ - expert criterion value; $v$ - criterion value in a unified system; $\alpha$ - reduction algorithm; $i$ - index of partial evaluation criterion.

Differences in v'and $\mathrm{v}$ indicator values determine the contents of the reduction algorithm, which in the simplest case may be expressed in a reduction rate (rate of v'to v) (e.g. in restating percentage points into fractions of a unit). Our experiments have led us to the conclusion that reduction with the help of relatively elementary algorithms are acceptable since they do not lose sight of precision which is needed in practice.

Evaluation of programme relevance is finished with calculating the value of a relative integrated criterion (7). The equation follows:

$$
T=\sum_{i=1}^{n} r_{i} v_{i} .
$$

\section{Evaluation of programme efficiency}

Evaluation of economic efficiency of programmes is a more complicated and more labour extensive exercise. We would like to propose a three-level evaluation of programmes, tasks and measures for fulfilling the tasks. It is noteworthy that alternatives arc possible at each level. The application of the principle of programme structure predetermines the logical order of evaluations in respect of items under evaluation from partial (measure) to the most general (programme). The logic here is easy to explain: each task (objectives of the task) may be fulfilled by different measures and their combinations. Consequently at this level (the lowest) determination of alternative measures and evaluation of their efficiency are certainly meaningful. At this stage labour costs are directly related to the number of alternatives and are in proportion with the number of alternative measures (variants).

Evaluation of efficiency at a task level is the same as evaluation at a measure level in methodological and technological terms (Fig. 3). The methodological specificity of these evaluations is as follows.

In step one the choice of evaluation criteria is predetermined by certain combinations of peculiarities of alternatives under evaluation. Our studies have identified four specific situations:

1) The output of alternatives being evaluated (measures or tasks) expressed in one single indicator;

2) The output of all the alternatives being evaluated expressed in several (more than 1) but the same indicators;

3) The output of the alternatives being evaluated expressed in one but not single indicator; 
4) The output of the alternatives being evaluated expressed in several (more than 1) indicators that are not the same for all the alternatives.

In the first instance evaluation is simplified as much as possible because a uniform criterion can be applied, then it being the benchmark, different options can be compared and the most efficient one chosen:

$$
e_{i}=\frac{R_{1}}{S}
$$

where: $R$ - value of the indicator expressing the output of measure implementation; $S$ - costs related to the implementation of measure.

The most efficient option is selected according to this equation:

$$
e=\max / \min \left\{e_{1}, e_{2}, \ldots, e_{n}\right\},
$$

and then either the maximum or the minimum value of the indicator means the highest efficiency.
The second instance differs in a necessity to grant significance (value of significance) to the criteria being applied to the package of which is determined by indicators that express alternative outcomes. That value should reflect the ratio of adjacent level elements (measure to task, task to programme), i.e. the impact of a lower level clement on the implementation of a higher level element, to be exact, the impact on the goal pursed by implementing it. The value is expressed in fractions of a unit under condition (1). Determination of significance of the criteria applied predetermines the necessity to calculate the values of an integrated efficiency indicator based on which (5) the most efficient option is selected.

Analytical calculations are not sufficient for evaluating alternatives in the third and fourth instances. The method of expert valuation (opinion) is applied by stating the efficiency of alternatives in relative points. In such cases in order to increase the objectivity of evaluations experts should be supplied with relative values of indicators and costs of each alternative that are calculated in advance according to equation (4).

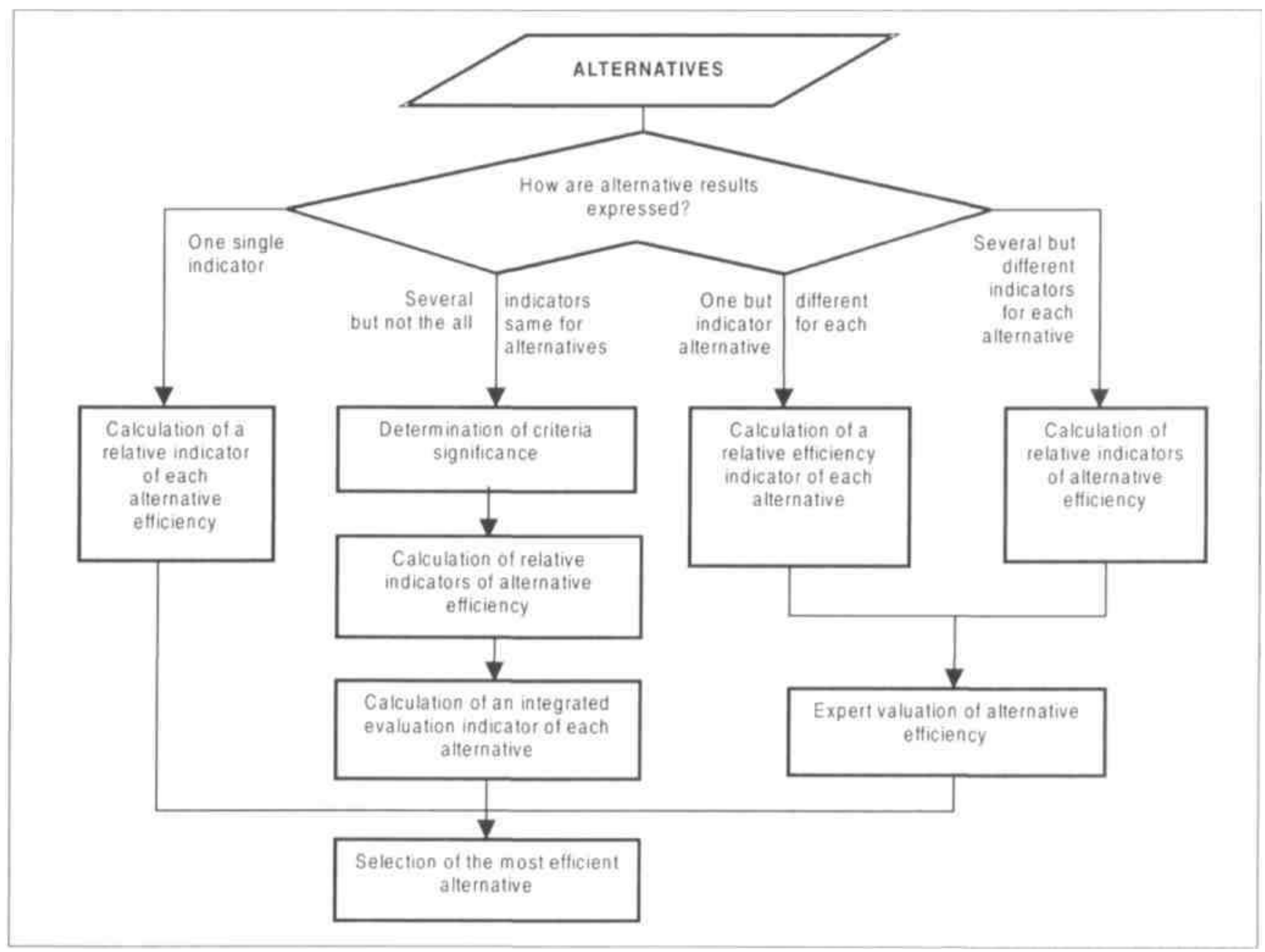

Fig 3. Technological sheme of programme evaluation on the level of measures and tasks 
The most complicated efficiency evaluation is conducted on a programme level. The complexity is conditioned by two factors:

1) Diversity of programmes, differences in goals and expressions of implementation outcomes (ranging from quantitative parameters to qualitative definitions), and sets of possible different combinations;

2) Need to benchmark programme.

Our experiments have shown that in view of the two factors above the most practically acceptable way is the calculation/evaluation of expected benefit (effect) of a programme (Fig. 4). The quantitative value of benefit is:

$$
E=d p
$$

where $d$ - relative efficiency of a programme; $p$ probability of relative efficiency of a programme.

A relative efficiency of a programme as well as the probability of getting it arc determined by a method of expert valuation (opinion). Relative efficiency of the programme is determined in points for the whole life cycle of the programme, i.e. for the period from the beginning of programme implementation to the end of benefit it produces. In order to ensure benchmarking possible margins of relative benefit points, for example, from 0 to 10 , should be set. The probabilities of getting benefit are set in a usual way, in fractions of a unit $\left(0^{\wedge} 1\right)$.

Expert valuation may be conducted by applying different methodologies, and the choice does not matter really, still the reliability of valuations may be increased by making use of several methods to be fol-

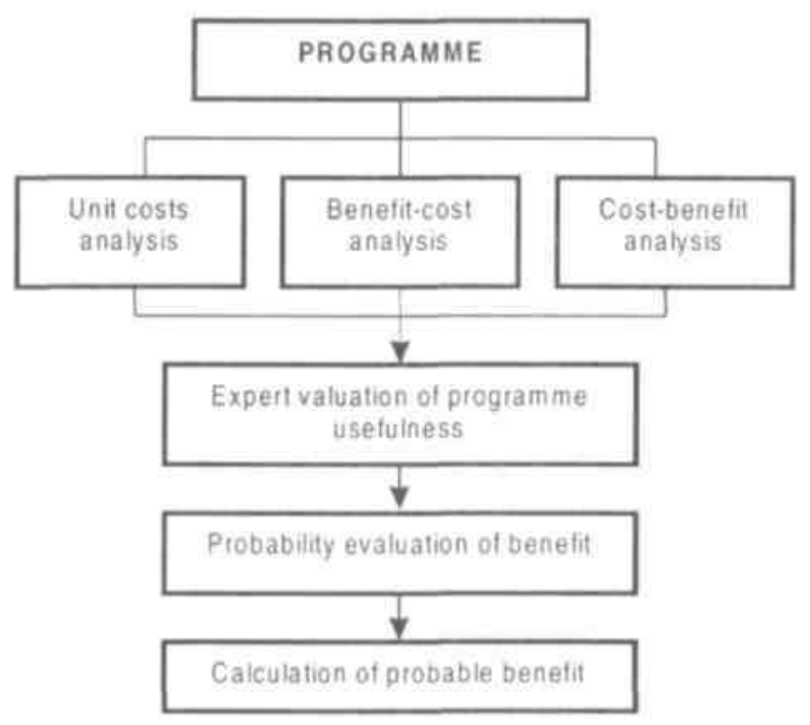

Fig 4. Principal scheme of programme efficiency lowed by the synthesis of the results obtained. Besides, the evaluation of as many as possible quantitative partial efficiency indicators may raise the reliability of expert valuations. We propose to use the following sufficiently universal indicators:

1) Unit costs (for each programme outcome expressed in quantitative terms):

$$
s_{i}=\frac{S}{R},
$$

where $S$ - total programme costs; $R$ - programme outcome expressed in quantitative terms; $\mathrm{j}$ - index of outcome expression;

$$
\tau_{j}=\frac{R_{i}}{S} \text {, }
$$

3) Cost-benefit:

$$
u=\frac{v}{s}
$$

\section{2) Benefit-cost:}

where $U$ - added value produced during the whole life cycle of a programme.

Discount values of both effect and costs should be sued. Time chosen for discounting values does not essentially matter, however bearing in mind the need of benchmarking, the most reasonable starting point should be the beginning of a budget period.

\section{Evaluation of programme fitness}

The third component of budget programme evaluation is the evaluation of its fitness, which is treated as being able to check and add to the two components mentioned above. In its essence, it is quality assessment where the preparation of a programme is evaluated in different respects (Fig 5). It is only reasonable to evaluate the fitness of the programme when relevance and efficiency results point to the acceptability of the programme. On the other hand, the values of partial fitness indicators may suggest the diagnosis of weaknesses of programme preparation. Their elimination may result in a higher quality of the programme.

Even though at first glance all the aspects of programme fitness evaluation are easily expressible in quantitative terms, our experiments have led us to the conclusion that such evaluations should comprise both analytical appraisals and expert valuations. Such expert valuations are needed in four initial evaluation stages (Fig 5), i.e. for determining a partial indicator 


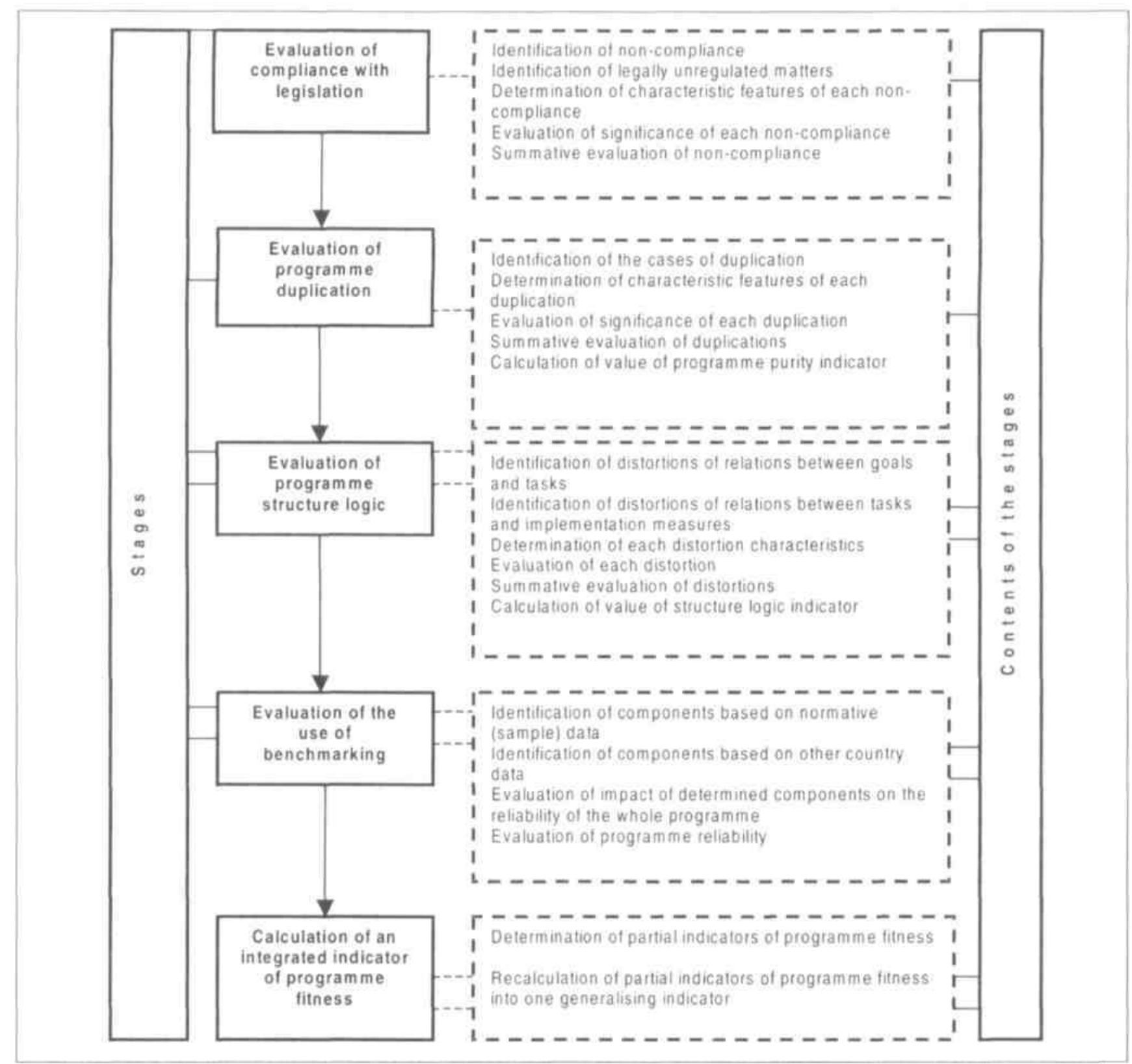

Fig 5. Principal scheme of the evaluation of programme fitness

of programme fitness. The need for expert valuations from different perspectives (non-compliance with legislation, duplication of programmes, distortions of programme structure logic, components based on the sameness of principles) is conditioned by the diversity of characteristic features of the cases being considered and the significance of situations described by such characteristic features to the fitness of the programme. The significance depends on all rather than only the considered characteristic features of the programme, as well as their relationships and different combinations:

$$
t=1-r
$$

where $r$ - value of totalling indicator of programme evaluation from a certain perspective.

Beside the values of partial fitness indicators the values of partial significance indicators are needed for getting at the value of integrated indicator for programme fitness. We suggest that the objectivity of benchmarking the programmes should be increased by setting the same unified (applicable to all the programmes) significance of partial fitness indicators in advance. For instance, our experiments conclude that 0.25 values attributed to each partial fitness indicator might be an acceptable solution. 


\section{Integrated complex evaluation}

Integrated complex evaluation of a programme is the synthesis of relevance, efficiency and fitness of the programme in view of the significance of each indicator and the values obtained for each of the indicators. The requirements for determination of integrated significance of

relevance, efficiency and fitness indicators for a programme follow:

1) Significance is expressed in relative values;

2) Relative values of significance are set under condition (1);

3) A unified system of significance values should by applied by all the appropriation managers to all the budget programmes prepared by them.

Bearing in mind the direct and decisive impact of significance values on final programme evaluation results, special attention should be paid to two most important evaluation factors - development of methodology and selection of experts. In this case the emphasis is on more reliable expert valuation methods, although this is a more labour extensive way. The formation of an expert team should be guided by competence of experts and their number that is determined on the basis quantitative appraisals.

When practical experience of budget programme evaluation is gained, the stage of evaluation in question will be fundamentally simplified to a mere adjustment of relative significance values or, maybe, to only expert checks.

The equation of getting an integrated evaluation indicator $(\mathrm{Z})$ is:

$$
\mathrm{Z}=\mathrm{r}, T+r_{2} E+r_{3} G
$$

where - relative values of significance of integrated programme relevance $(r$,$) , efficiency (r \sim$, ) and fitness $\left(\mathrm{r}_{3}\right)$ indicators; $T$ - value of an integrated programme relevance indicator; $E$ - value of an integrated programme efficiency indicator; $G$ - value of an integrated programme fitness indicator.

Continuous programmes should be evaluated differently than programme drafts (new programmes). We propose a different way of evaluating the efficiency of programme implementation. In terms of contents, it is a two-stage analysis. An actual integrated indicator of the programme implementation efficiency is the first stage of the evaluation, while the second covers the comparison of the actual indicator value with the designed value that is followed by the situation analysis of the programme implementation. The aim of the second stage is twofold: determination of the degree of implementation of different programme components (tasks, measures) and appraisal of the impact of fully and partially implemented measures on the integrated efficiency indicator.

\section{Conclusions}

1. The allocation of public financial resources is one of the key tasks of public administration. What creates preconditions for more efficient use of the most significant resources, delivery of state priorities and invigoration of activities in identified areas is rational allocation of these resources.

2. Despite recent constructive innovations of the methods of allocation of public financial resources, the following shortcomings arc still typical of dealing with the task: absence of rules of allocation of financial resources, doubtful and unreasonable criteria, or inability to apply them.

3. A model of budget programme evaluation based on the principle of integrated evaluation and comprised of four main evaluation components: programme relevance, programme efficiency, programme fitness and integrated evaluation of each programme is proposed. Each of the first three components are designed to evaluate a budget programme from a certain perspective, while the fourth one is the synthesis of the three evaluation results.

4. The application of the proposed programme evaluation model should result in the following:

a) quantitative programme benchmarking would increase the objectivity of programme evaluation and reduce the influence of subjective factors;

b) preconditions for benchmarking different institutions and programmes would be created;

c) debates on budget programmes and their assessment in the budgeting process would be simplified, and unproductive labour costs in relation to debates on programme drafts by different authorities (appropriation managers, Ministry of Finance, the Cabinet, and the Prime Minister's Office, parliamentary committees and party groups) would be reduced;

d) the process of budgeting would become more transparent and better understandable to the publie;

e) an economic effect due to more reasonable allocation of public financial resources would be achieved. 


\section{References}

1. Bivainis J., Butkevičius A. The National Budget Expenditure Planing // Monetary Studies (Nacionalinio biudžeto išlaidų planavimas // Pinigų studijos). 2002, No 4. p. 5-20 (in Lithuanian).

2. Budgeting and Policy Making. SIGMA Papers: No 8. Paris: OECD. 1996. 335 p.

3. Potter B. H., Diamond J. Guidelines for Public Expenditure Management. IMF. 1999. 69 p.

4. Premchand A. Government Budgeting and Expenditure Controls: Theory and Practice. IMF. 1994. 350 p.

5. Budgeting for the Future. Paris: OECD. Vol. 5, No 95. 1997. $25 \mathrm{p}$

6. Likierman A. Changes to Managerial Decision-Taking in UK Central Government //Management Accounting Research. 2000, Vol. 11, No 2, p 253-261.
7. Modern Budgeting. Paris: OECD. 1997. 134 p.

8. Managing Public Expenditure. A Reference Book for Transition Countries // Edited by R. Allen and D. Tommasi. OECD. 2001. 500 p.

9. Model S. Performance Measurement and Institutional Processes: a Study of Managerial Responses to Public Sector Reform // Management Accounting Research. 2001, Vol. 12, No 4, p. 437-464.

10. Ex ante Evaluation. A Practical Guide for Preparing Proposals for Expenditure Programmes. European Commission. December 2001. 29 p.

11. Evaluating EU Expenditure Programmes: A Guide. Ex post and Intermediate Evaluation. European Commission. First edition, January 1997. 93 p. 